POS PROCEEDINGS

\title{
Energy dependence of anisotropic flow
}

\author{
Raimond Snellings \\ NIKHEF \\ E-mail: Raimond.Snellings@nikhef.nl
}

These proceedings give a brief selective overview of anisotropic flow at RHIC. In addition an outlook on the feasability of studing anisotropic flow at the LHC is given.

The 3rd edition of the International Workshop — The Critical Point and Onset of Deconfinement July 3-7 2006

Galileo Galilei Institute, Florence, Italy 


\section{Introduction}

Flow is observed in nucleus-nucleus collisions from low energies up to $\sqrt{s_{\mathrm{NN}}}=200 \mathrm{GeV}$ at the Relativistic Heavy Ion Collider (RHIC), and is expected to be observed at the Large Hadron Collider (LHC). Flow signals the presence of multiple interactions between the constituents and is an unavoidable consequence of thermalization.

The usual theoretical tools to describe flow are hydrodynamic or microscopic transport (cascade) calculations. Flow depends in the transport models on the opacity, be it partonic or hadronic. Hydrodynamics becomes valid when the mean free path of particles is much smaller than the system size and allows for a description of the system in terms of macroscopic quantities. This gives a handle on the equation of state of the flowing matter and, in particular, on the value of the sound velocity [1]. In both types of models it may be possible to deduce from a flow measurement whether the flow originates from partonic or hadronic matter or from the hadronization process $[2,3,4]$.

A convenient way of characterizing the various patterns of anisotropic flow is to use a Fourier expansion of the triple differential invariant distributions [5]:

$$
E \frac{\mathrm{d}^{3} N}{\mathrm{~d}^{3} \mathbf{p}}=\frac{1}{2 \pi} \frac{\mathrm{d}^{2} N}{p_{\mathrm{t}} \mathrm{d} p_{\mathrm{t}} \mathrm{d} y}\left\{1+2 \sum_{n=1}^{+\infty} v_{n} \cos \left[n\left(\varphi-\Psi_{R}\right)\right]\right\},
$$

where $\varphi$ and $\Psi_{R}$ are the particle and reaction-plane azimuths in the laboratory frame, respectively. The sine terms in such an expansion vanish due to reflection symmetry with respect to the reaction plane. The Fourier coefficients are given by

$$
v_{n}\left(p_{\mathrm{t}}, y\right)=\left\langle\cos \left[n\left(\varphi-\Psi_{R}\right)\right]\right\rangle,
$$

where the angular brackets denote an average over the particles, summed over all events, in the $\left(p_{\mathrm{t}}, y\right)$ bin under study. In this parameterization, the first two coefficients, $v_{1}$ and $v_{2}$, are known as directed and elliptic flow, respectively.

\section{Elliptic Flow: $v_{2}$}

Elliptic flow has its origin in the amount of rescattering and the spatial eccentricity of the collision zone. The amount of rescattering is expected to increase with increasing centrality, while the spatial eccentricity decreases. This combination of trends dominates the centrality dependence of elliptic flow. The spatial eccentricity is defined by

$$
\varepsilon=\frac{\left\langle y^{2}-x^{2}\right\rangle}{\left\langle y^{2}+x^{2}\right\rangle}
$$

where $x$ and $y$ are the spatial coordinates in the plane perpendicular to the collision axis. The brackets \langle\rangle denote an average weighted with the initial density. Generally speaking, large values of elliptic flow are considered signs of hydrodynamic behavior as was first put forward by Ollitrault [1]. In hydrodynamics $v_{2}$ is essentially proportional to the spatial eccentricity (the strength depends on the velocity of sound).

Figure 1 shows the first measurement of elliptic flow at RHIC [6]. The open rectangles in Fig. 1 show, for a range of possible values of the velocity of sound, the expected $v_{2}$ values from 


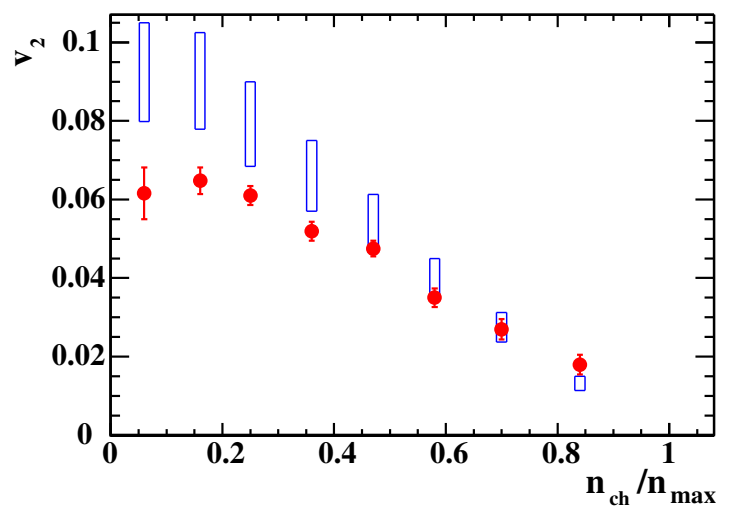

Figure 1: Elliptic flow (solid points) as a function of centrality defined as $n_{c h} / n_{\max }$. The open rectangles show a range of values expected for $v_{2}$ in the hydrodynamic limit, scaled from $\varepsilon$, the initial space eccentricity of the overlap region. From [6]

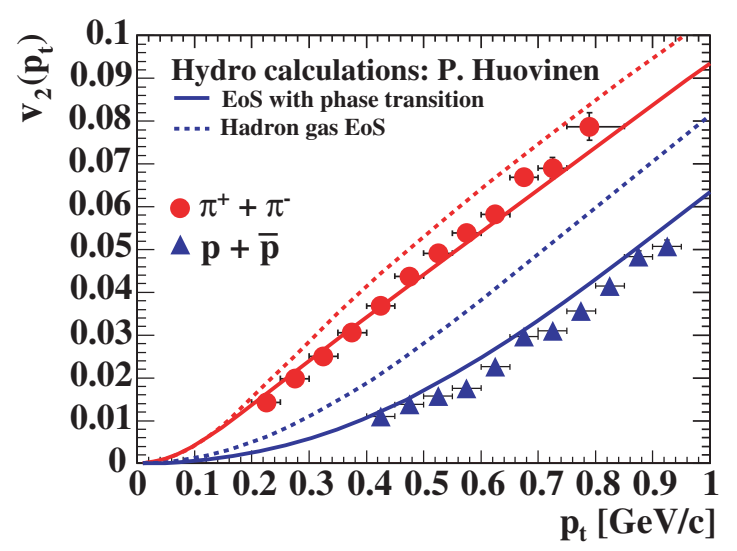

Figure 2: Elliptic flow of pions and protons as function of transverse momentum [12]. The lines are hydrodynamical model calculations using two different Equations of State (EoS).

ideal hydrodynamics. For $n_{c h} / n_{\max } \geq 0.5$ (b $\leq 7 \mathrm{fm}$ ) it is observed that the data is well described by ideal hydrodynamics. The observed large amount of collective flow, in particular elliptic flow, is one of the main experimental discoveries at RHIC $[7,8,9,10]$ and the main evidence suggesting nearly perfect fluid properties of the created matter [11].

Figure 2 shows $v_{2}$ for identified particles as function of transverse momentum. At low $p_{\mathrm{t}}$ the elliptic flow depends on the mass of the particle with $v_{2}$ at a fixed $p_{\mathrm{t}}$ decreasing with increasing mass. This dependence is expected in a scenario where all the particles have a common radial flow velocity $[13,14]$ as shown by the curves in Fig. 2 from ideal hydrodynamics. The difference between the dashed and solid curves is the EoS, the dashed curves correspond to calculations done with a hadron resonance gas EoS while the solid curves are hydro calculations incorporating the QCD phase transition. The sensitivity to the EoS is better for the heavier particles because they are less affected by the contribution of the finite freeze-out temperature. It is clear that the hydro calculations incorporating the QCD phase transition give better description of the observed mass splitting. However, detailed constrains on the EoS can only be obtained with better modeling of the hadronic phase $[15,16,17,18]$ and the transition [19] between the QGP and hadronic phase.

In ideal hydrodynamics the mass ordering in $v_{2}$ persists up to arbitrary large $p_{\mathrm{t}}$, although less pronounced because the $v_{2}$ of the different particles start to approach each other. Figure 3 shows that at higher $p_{\mathrm{t}}$ the measurements start to deviate significantly from hydrodynamics for all particle species, and that the observed $v_{2}$ of the heavier baryons is larger than that of the lighter mesons. This mass dependence is the reverse of the behavior observed at low $p_{\mathrm{t}}$. This is not expected in hydrodynamics and is also not expected if the $v_{2}$ is caused by parton energy loss (in the latter case there would, to first order, be no particle type dependence). An elegant explanation of the unexpected particle type dependence and magnitude of $v_{2}$ at large $p_{\mathrm{t}}$ is provided by the coalescence picture. In the coalescence picture the $v_{2}$ scales, instead of particle mass, with the number of constituent quarks and this is considered evidence for the partonic origin of flow [22, 23].

The agreement between the measured $v_{2}$ and ideal hydrodynamics calculations at RHIC seems 


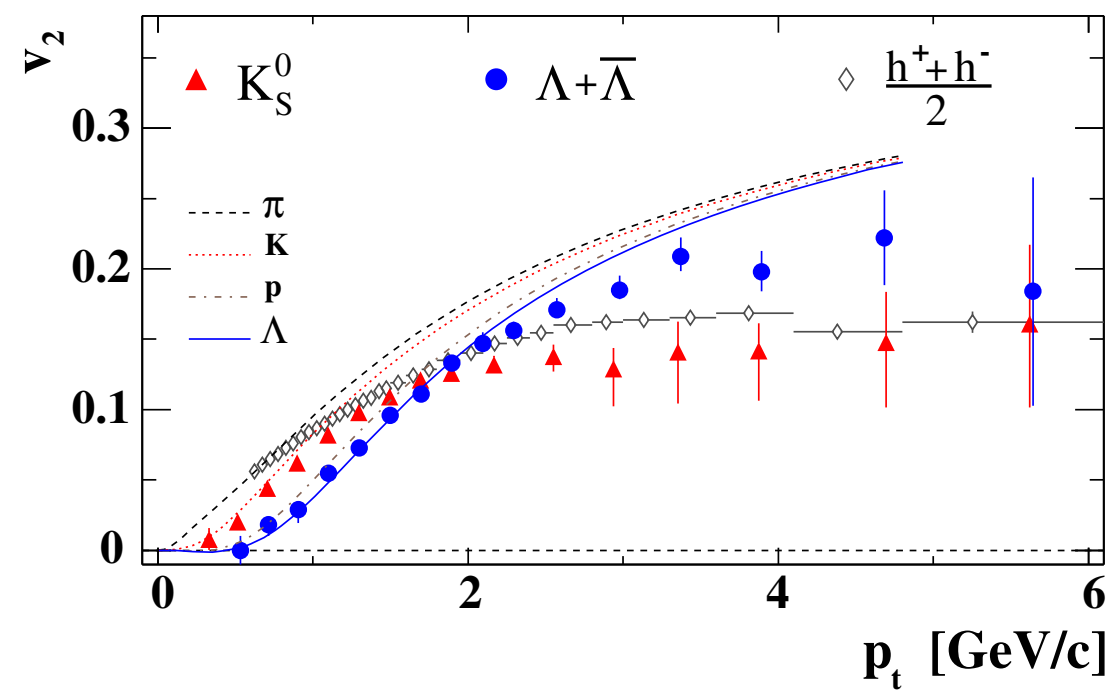

Figure 3: The minimum-bias (0-80\% of the collision cross section) $v_{2}\left(p_{T}\right)$ for $K_{S}^{0}, \Lambda+\bar{\Lambda}$ and $h^{ \pm}$. Hydrodynamical calculations of $v_{2}$ for pions, kaons, protons and lambdas are also plotted [20]. From [21]

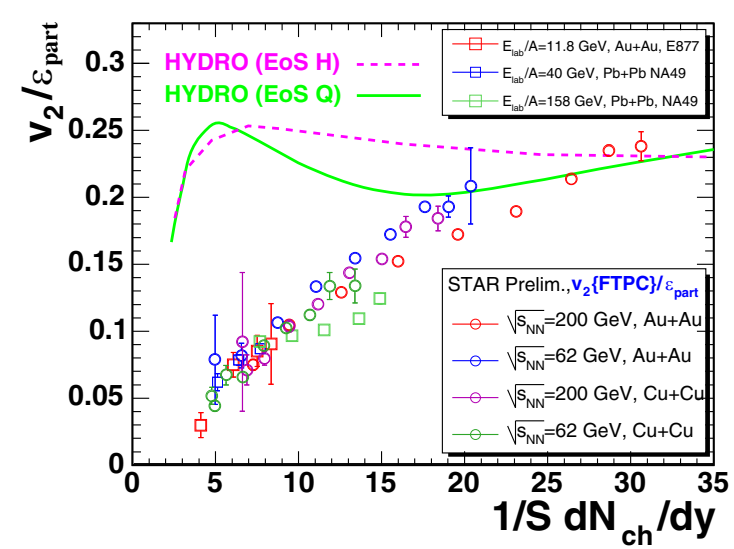

Figure 4: $v_{2} / \varepsilon_{\text {part }}$ from STAR for $\mathrm{Au}+\mathrm{Au}$ and $\mathrm{Cu}+\mathrm{Cu}$ collisions at 62.4 and $200 \mathrm{GeV}$, combined with measurements from NA49 and E877 at SPS and AGS energies, respectively. From [24].

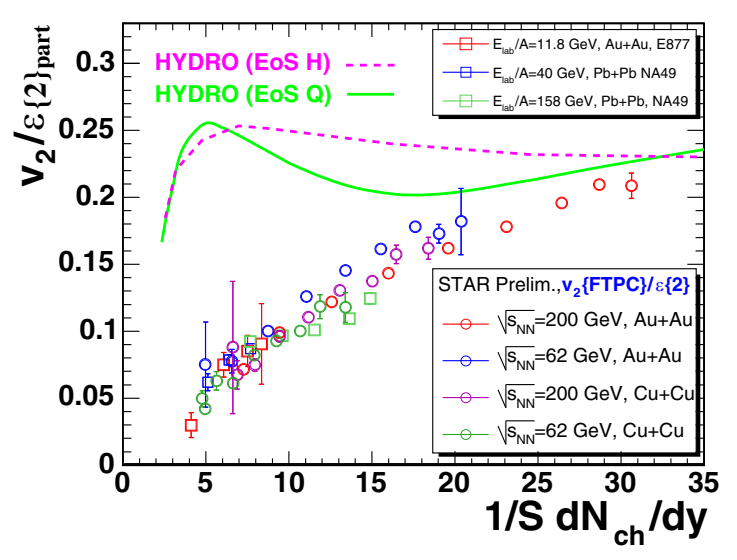

Figure 5: $v_{2} / \varepsilon\{2\}$ part from STAR for $\mathrm{Au}+\mathrm{Au}$ and $\mathrm{Cu}+\mathrm{Cu}$ collisions at 62.4 and $200 \mathrm{GeV}$, combined with measurements from NA49 and E877 at SPS and AGS energies, respectively. From [24].

to indicate that the matter created behaves like an perfect fluid. To better understand the perfect fluid behavior one should study elliptic flow as function of collision system, beam energy and centrality. The collision system, energy and centrality dependence can be combined by plotting $v_{2} / \varepsilon$ as function of $1 / S \mathrm{~d} N_{c h} / \mathrm{d} y$, where $S$ is a measure of the initial transverse size of the collision region [25, 26, 27]. It was shown by the PHOBOS collaboration [28] that fluctuations in the nucleon positions, particularly in small systems and for small eccentricities, create a situation where the minor axis of the ellipse is not along the impact parameter vector. This can be addressed by a rotation of the $x$ and $y$ axis such that $\left\langle x^{2}\right\rangle$ is minimized [28]. The spatial participant eccentricity is 
defined by

$$
\varepsilon_{\text {part }}=\frac{\left\langle y^{\prime 2}-x^{\prime 2}\right\rangle}{\left\langle y^{\prime 2}+x^{\prime 2}\right\rangle}
$$

where $x^{\prime}$ and $y^{\prime}$ are now the spatial coordinates in the rotated plane perpendicular to the collision axis $[28,29,30]$. Figure 4 shows the $p_{\mathrm{t}}$-integrated $v_{2}$ divided by the spatial eccentricity $\varepsilon_{\text {part }}$ as a function of the multiplicity density. It is seen that $v_{2} / \varepsilon_{\text {part }}$ falls approximately on a single curve, independent of collision system, beam energy and impact parameter. This curve shows an approximately linear increase from AGS to the highest RHIC energy. Ideal hydrodynamical model calculations, whose elliptic flow predictions rather well matched the measurements at the highest RHIC energies, show a markedly different behavior. In hydrodynamics, $v_{2} / \varepsilon$ depends on the velocity of sound in the fluid and thus on the different contributions from the different phases and the phase transition. The hadronic equation of state (EoS H in Fig. 4) with a fixed velocity of sound shows therefore an almost constant $v_{2} / \varepsilon$. The equation of state incorporating the QCD phase transition (EoS Q in Fig. 4) shows a more complicated energy dependence. At AGS and SPS energies EoS Q is dominated by the rather large velocity of sound of the hadronic phase, at RHIC energies the system spends a significant part of its evolution in the mixed phase which causes the drop in the velocity of sound and thus in the $v_{2}$, at higher energies the partonic phase starts to dominate the system evolution which increases the effective velocity of sound and thus $v_{2}$ again. The disagreement between the data and hydrodynamical calculations at lower energies is interpreted as a sign of incomplete thermalization. Indeed, models that assume a mean free path of the same order as the size of the system (the so-called low density limit) show a monotonic dependence of $v_{2} / \varepsilon$ versus $1 / S \mathrm{~d} N / \mathrm{d} y$ as seen in the data $[25,26,27]$.

Since the $v_{2}$ in Fig. 4 is calculated from $\sqrt{\left\langle v_{2}^{2}\right\rangle}$ and $v_{2} \propto \varepsilon$ it is natural to also calculate $\varepsilon_{\text {part }}$ from $\sqrt{\left\langle\varepsilon_{\text {part }}^{2}\right\rangle}[29,30,31]$. Following the notation from [31] this eccentricity is denoted by $\varepsilon\{2\}_{\text {part }}$. Figure 5 shows the $v_{2} / \varepsilon\{2\}_{\text {part }}$ as function of the multiplicity density [24], this scaling indeed works somewhat better than the scaling by $\varepsilon_{\text {part }}$ as was shown in Fig. 4.

\section{Higher Harmonics}

The higher harmonics of the momentum anisotropy are generally expected to be small $[32,33]$. It was realized that at higher $p_{\mathrm{t}}$ they may become significant [34], which renewed experimental interest [35]. With the recent realization that the ratio of $v_{4} / v_{2}^{2}$ is a sensitive probe for ideal hydrodynamical behavior and thermalization, measuring these higher harmonics has clearly become an important tool $[27,36]$. It is shown in [36] that for an ideal fluid the ratio $v_{4}\left(p_{\mathrm{t}}\right) / v_{2}^{2}\left(p_{\mathrm{t}}\right)$ should approach 0.5 .

The measured $p_{\mathrm{t}}$-integrated $v_{4}$ and $v_{6}$ as function of centrality are shown in Fig. 6 [35]. For comparison, $v_{2}$ is shown in the same figure. The integrated $v_{4}$ is an order of magnitude smaller than $v_{2}$, as expected. The higher harmonics $v_{6}$ and $v_{8}$ (not shown) are consistent with zero. The upper dashed line in Fig. 6 shows that the ratio $v_{4} / v_{2}^{2}$ is larger than unity for all centralities which seems to contradict the prediction from ideal fluid behavior. However Refs. [27, 36] show that this asymptotic value of $v_{4}=v_{2}^{2} / 2$ is reached only at transverse momenta well above $1 \mathrm{GeV} / \mathrm{c}$. At RHIC the ratio of $p_{\mathrm{t}}$-integrated $v_{4} / v_{2}^{2}$ is mainly determined by particles below $1 \mathrm{GeV} / c$. 


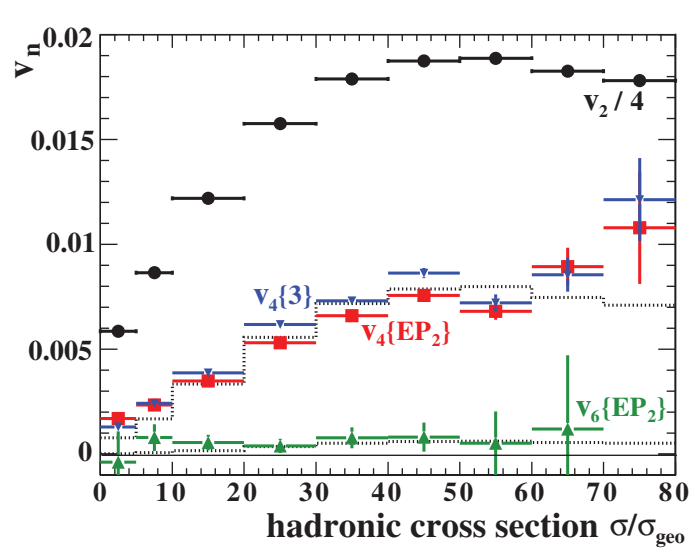

Figure 6: The $p_{t}$ - and $\eta$ - integrated values of $v_{2}$, $v_{4}$, and $v_{6}$ as a function of centrality. The dotted histograms are $1.4 \cdot v_{2}^{2}$ and $1.4 \cdot v_{2}^{3}$. In $v_{n}\{\}$ the term in the curly brackets indicates the method used [37]. Figure from [35].

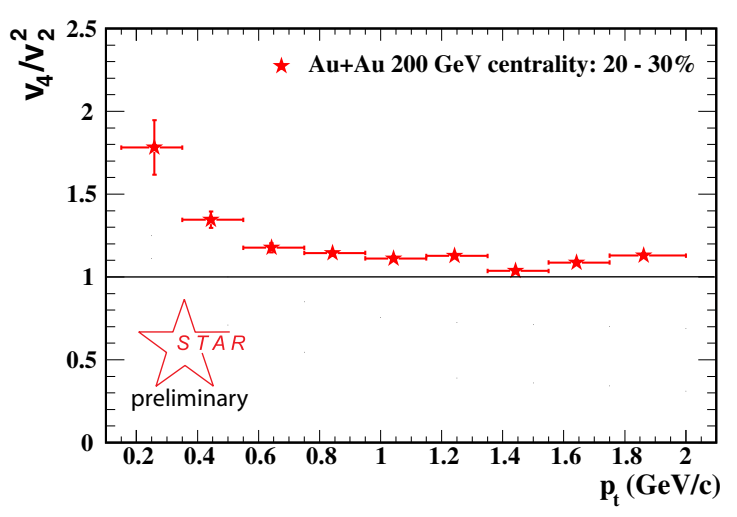

Figure 7: $v_{4}\left\{\mathrm{EP}_{2}\right\} / v_{2}^{2}\{4\}$ in $\mathrm{Au}+\mathrm{Au}$ collisions at $\sqrt{s_{N N}}=200 \mathrm{GeV}[38]$.

A more detailed comparison is the transverse momentum dependence of $v_{4} / v_{2}^{2}$ as shown in Fig. 7. It seen that the general $p_{\mathrm{t}}$-dependence is in agreement with hydro expectation. However the minimum of $v_{4} / v_{2}^{2}$ is still more than a factor of two larger than the asymptotic ideal hydro value of 0.5. Measurements of the energy dependence of this ratio, particularly at an order of magnitude higher beam energy at the LHC, should provide insight into the dynamics driving this ratio [27].

\section{Anisotropic flow at the Large Hadron Collider}

With the models which successfully describe the properties of the matter created at RHIC one can (and should) make predictions for the LHC. Testing these predictions will provide important confirmation of, or perhaps new insights to, our current understanding of QCD matter. Figure 8 shows elliptic flow calculations for the LHC. Using Color Glass Condensate (CGC) estimates for the initial energy density, the flow is calculated using ideal hydrodynamics up to the kinematic freeze-out temperature of $100 \mathrm{MeV}$ (full squares and upper curve) [39]. More realistic estimates are obtained by assuming hydrodynamics up to the chemical freeze-out temperature of $169 \mathrm{MeV}$ followed by a hadron cascade description of the final phase (full circles). The contribution from the QGP phase (i.e. hydrodynamics up to $169 \mathrm{MeV}$ ) is shown by the triangles (and lower curve) in the figure. It is seen from Fig. 8 that at LHC energies the contribution from the QGP phase is much larger than at RHIC or SPS, and that, as a consequence, there is less uncertainty due to the detailed modeling of the hadronic phase. Theoretical calculations such as these or e.g. in Ref. [40], as well as straight-forward extrapolations from lower energies based on particle multiplicities predict maximum flow values of about 5-10\% at the LHC. If the flow values (and corresponding multiplicities) at the LHC are indeed that large then the flow measurement should be relatively easy.

The previous hydro estimates assume that during the QGP phase the matter has zero shear viscosity. Teaney [41] has shown that even a small shear viscosity has a large effect on the buildup of flow. Recent calculations [17, 42] show how the viscosity increases from RHIC to the LHC. To 


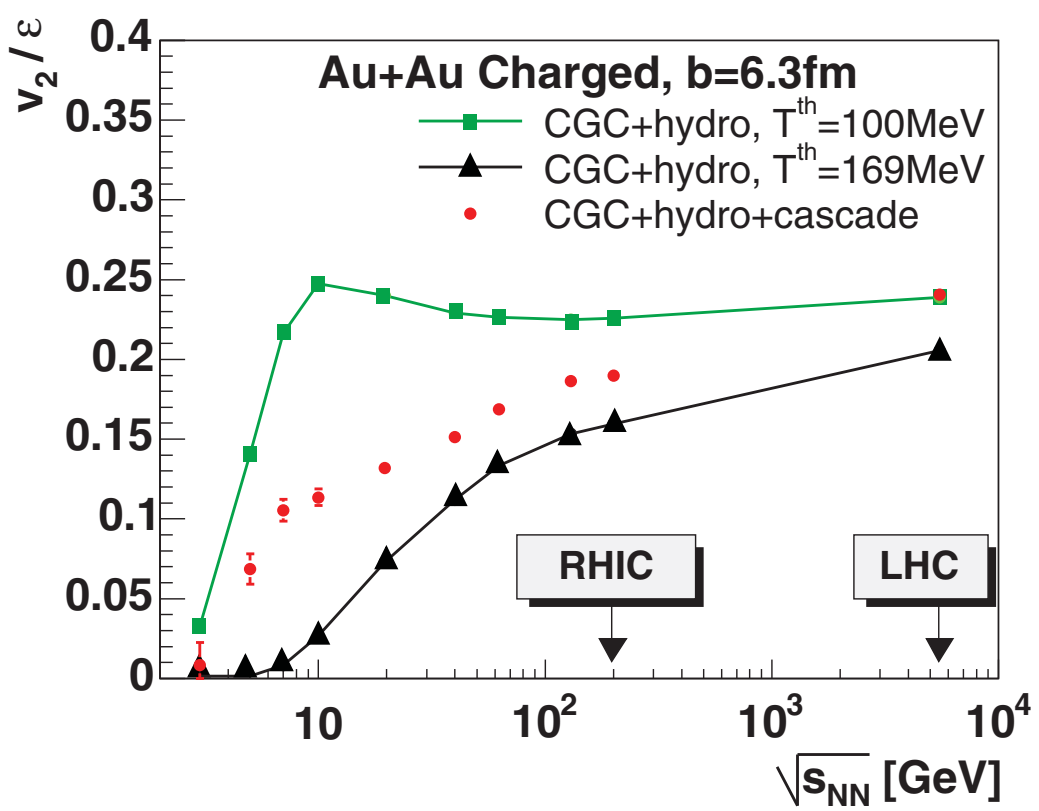

Figure 8: Theoretical predictions [39] of $v_{2} / \varepsilon$ versus collision energy using color glass condensate estimates for the initial energy density. Ideal hydrodynamic expansion up to kinetic freeze-out (squares) or chemical freeze-out (triangles) is assumed. The full circles are results using a hadronic cascade model to describe the final phase after chemical freeze-out.

estimate how this would affect the predicted flow, viscous corrections have to be implemented in hydro models [43].

In addition, as mentioned before, experimental measurements of flow are affected by biases from physical effects unrelated to anisotropic flow ('non-flow effects' like e.g. jet correlations) or due to additional features of the flow signal itself (e.g. fluctuations [31, 29, 30, 44]). To estimate the effect of jet like correlations at the LHC a simple estimate can be made similar to what was done for the first RHIC flow measurement [6]. The estimate of the non-flow is given by

$$
\left\langle\cos \left[n\left(\Psi_{2}^{a}-\Psi_{2}^{b}\right)\right]\right\rangle \propto M_{\text {sub }} v_{2}^{2}+\tilde{g},
$$

where the angular brackets denote an average over the events, $\Psi_{2}^{a, b}$ are the subevent event planes, $M_{\text {sub }}$ is the corresponding subevent multiplicity and $\tilde{g}$ is the non-flow component. The estimated value of $\tilde{g}$ from HIJING at $\sqrt{s_{N N}}=130 \mathrm{GeV}$ in the STAR acceptance using random subevents was 0.05. In the ALICE TPC acceptance the value of $\tilde{g}$ from HIJING at $\sqrt{s_{N N}}=5.5 \mathrm{TeV}$ is found to be 0.08 . With better tuned definitions of the subevents the value of $\tilde{g}$ could easily be reduced to 0.04 . The correlation due to flow, $M_{\text {sub }} v_{2}^{2}$, is expected to be much larger than the non-flow contribution, 0.04 , in a large centrality range for $\mathrm{Pb}+\mathrm{Pb}$ collisions measured by ALICE at the LHC. This then indeed suggest that measuring flow can be done in great detail at the LHC.

\section{Conclusions}

At RHIC the observed large elliptic flow provides compelling evidence for strongly interacting 
matter which, in addition, appears to behave like an almost ideal fluid [11]. For the measurements, the system size, energy and centrality dependence of elliptic flow collapses to a single curve when plotted versus $1 / S \mathrm{~d} N / \mathrm{d} y$. Predictions for an ideal fluid for the system size, energy and centrality dependence are markedly different. This disagreement between the data and hydrodynamical calculations is interpreted as a sign of incomplete thermalization at lower energies. Compared to $v_{2}$ the ratio $v_{4} / v_{2}^{2}$ is argued to be even more sensitive to ideal hydro behavior and thermalization. At low $p_{\mathrm{t}}$ the ratio $v_{4} / v_{2}^{2}$, exhibits the transverse momentum dependence expected for an ideal fluid. However, the magnitude of $v_{4} / v_{2}^{2}$ is still more than a factor of two larger than the asymptotic ideal hydro value, which even at the highest RHIC energies might indicate incomplete thermalization. At the LHC the expected increase in multiplicity together with the expected increase in anisotropic flow will allow for a detailed measurement of the $v_{2}$ and higher harmonics [45]. These measurements at the LHC are expected to quickly provide important confirmations, or perhaps new insights to our current understanding of the EoS of QCD matter.

\section{References}

[1] J. Y. Ollitrault, Phys. Rev. D 46 (1992) 229.

[2] P. Danielewicz, Nucl. Phys. A 661 (1999) 82.

[3] D. H. Rischke, Nucl. Phys. A 610 (1996) 88C.

[4] J. Y. Ollitrault, Nucl. Phys. A 638 (1998) 195.

[5] S. Voloshin and Y. Zhang, Z. Phys. C 70 (1996) 665.

[6] K. H. Ackermann et al. [STAR Collaboration], Phys. Rev. Lett. 86 (2001) 402

[7] I. Arsene et al. [BRAHMS Collaboration], Nucl. Phys. A 757 (2005) 1

[8] B. B. Back et al. [PHOBOS Collaboration], Nucl. Phys. A 757 (2005) 28

[9] J. Adams et al. [STAR Collaboration], Nucl. Phys. A 757 (2005) 102

[10] K. Adcox et al. [PHENIX Collaboration], Nucl. Phys. A 757 (2005) 184

[11] T.D. Lee et al., New Discoveries at RHIC: Case for the Strongly Interacting Quark-Gluon Plasma. Contributions from the RBRC Workshop held May 14-15, 2004. Nucl. Phys. A 750 (2005) 1-171

[12] C. Adler et al. [STAR Collaboration], Phys. Rev. Lett. 87 (2001) 182301

[13] N. Borghini, P. M. Dinh and J. Y. Ollitrault, arXiv:hep-ph/0111402.

[14] J. P. Blaizot, Nucl. Phys. A 698 (2002) 360.

[15] D. Teaney, J. Lauret and E. V. Shuryak, arXiv:nucl-th/0110037.

[16] D. Teaney, J. Lauret and E. V. Shuryak, Phys. Rev. Lett. 86 (2001) 4783.

[17] T. Hirano and M. Gyulassy, Nucl. Phys. A 769 (2006) 71

[18] T. Hirano, U. W. Heinz, D. Kharzeev, R. Lacey and Y. Nara, arXiv:nucl-th/0511046.

[19] P. Huovinen, Nucl. Phys. A 761 (2005) 296

[20] P. Huovinen, P. F. Kolb, U. W. Heinz, P. V. Ruuskanen and S. A. Voloshin, Phys. Lett. B 503 (2001) 58

[21] J. Adams et al. [STAR Collaboration], Phys. Rev. Lett. 92 (2004) 052302 
[22] S. A. Voloshin, Nucl. Phys. A 715 (2003) 379.

[23] D. Molnar and S. A. Voloshin, Phys. Rev. Lett. 91, 092301 (2003)

[24] S. A. Voloshin [STAR Collaboration], AIP Conf. Proc. 870 (2006) 691

[25] H. Heiselberg and A. M. Levy, Phys. Rev. C 59 (1999) 2716

[26] S. A. Voloshin and A. M. Poskanzer, Phys. Lett. B 474 (2000) 27

[27] R. S. Bhalerao et al., Phys. Lett. B 627 (2005) 49.

[28] S. Manly et al. [PHOBOS Collaboration], Nucl. Phys. A 774 (2006) 523

[29] S. A. Voloshin, arXiv:nucl-th/0606022.

[30] R. S. Bhalerao and J. Y. Ollitrault, arXiv:nucl-th/0607009.

[31] M. Miller and R. Snellings, arXiv:nucl-ex/0312008.

[32] P. F. Kolb, J. Sollfrank and U. W. Heinz, Phys. Lett. B 459 (1999) 667.

[33] D. Teaney and E. V. Shuryak, Phys. Rev. Lett. 83 (1999) 4951.

[34] P. F. Kolb, Phys. Rev. C 68 (2003) 031902.

[35] J. Adams et al. [STAR Collaboration], Phys. Rev. Lett. 92 (2004) 062301

[36] N. Borghini and J. Y. Ollitrault, arXiv:nucl-th/0506045.

[37] N. Borghini, P. M. Dinh and J. Y. Ollitrault, Phys. Rev. C 64 (2001) 054901

[38] A.H. Tang [for the STAR Collaboration], arXiv:nucl-ex/0608026

Y. Bai, 2006 RHIC \& AGS Annual Users’ Meeting, http://www.bnl.gov/rhic_ags/users_meeting/Workshops/8.asp

[39] T. Hirano, Talk given at the Workshop on QGP Thermalization (QGPTH05), Vienna, Private communication.

[40] P. F. Kolb, J. Sollfrank and U. W. Heinz, Phys. Rev. C 62 (2000) 054909.

[41] D. Teaney, Phys. Rev. C 68 (2003) 034913

[42] L. P. Csernai, J. I. Kapusta and L. D. McLerran, arXiv:nucl-th/0604032.

[43] U. W. Heinz, arXiv:nucl-th/0512049.

[44] B. Alver [the PHOBOS Collaboration], arXiv:nucl-ex/0608025.

[45] F. Carminati et al. [ALICE Collaboration], J. Phys. G 30 (2004) 1517.

B. Alessandro et al. [ALICE Collaboration], J. Phys. G 32 (2006) 1295. 